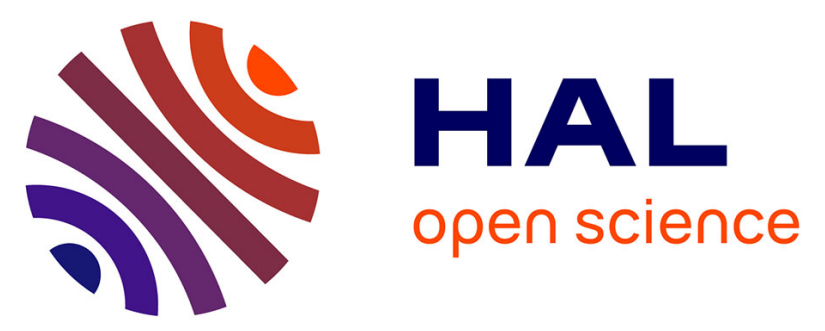

\title{
Etude de l'évolution des caractéristiques physico-chimiques et de la stabilité biologique des ordures ménagères au cours du compostage
}

Jean-Louis Morel, Armand Guckert, Bernard Nicolardot, Daniel Benistant, Gérard Catroux, Jean-Claude Germon

\section{To cite this version:}

Jean-Louis Morel, Armand Guckert, Bernard Nicolardot, Daniel Benistant, Gérard Catroux, et al.. Etude de l'évolution des caractéristiques physico-chimiques et de la stabilité biologique des ordures ménagères au cours du compostage. Agronomie, 1986, 6 (8), pp.693-701. hal-00884926

\section{HAL Id: hal-00884926 \\ https://hal.science/hal-00884926}

Submitted on 1 Jan 1986

HAL is a multi-disciplinary open access archive for the deposit and dissemination of scientific research documents, whether they are published or not. The documents may come from teaching and research institutions in France or abroad, or from public or private research centers.
L'archive ouverte pluridisciplinaire HAL, est destinée au dépôt et à la diffusion de documents scientifiques de niveau recherche, publiés ou non, émanant des établissements d'enseignement et de recherche français ou étrangers, des laboratoires publics ou privés. 


\title{
Etude de l'évolution des caractéristiques physico- chimiques et de la stabilité biologique des ordu- res ménagères au cours du compostage
}

\author{
Jean-Louis MOREL, Armand GUCKERT, Bernard NICOLARDOT $\left({ }^{*}\right)$, Daniel BENISTANT $\left({ }^{*}\right)$, \\ Gérard CATROUX $\left({ }^{*}\right)$ \& Jean-Claude GERMON $\left({ }^{*}\right)$ \\ E.N.S.A.I.A., 2, avenue de la Forêt de Haye, F 54500 Vandoeuvre \\ (*) I.N.R.A., Laboratoire de Microbiologie des Sols, 17, rue Sully, F 21034 Dijon Cedex
}

RÉSUMÉ

\begin{abstract}
Une étude analytique a été conduite sur un échantillonnage de composts urbains et d'ordures broyées prélevés à des âges différents sur 14 usines ou décharges situées en France. Les analyses ont porté sur des paramètres physiques et chimiques. On a suivi d'autre part certaines fractions organiques impliquées dans le compostage (hydrates de carbòne, composés alcalino-solubles). Enfin, des expériences de respirométrie ont été conduites afin de déterminer la stabilité biologique des composts.

Initialement, les ordures ménagères fraîches présentent des compositions très variables. Leur évolution conduit à des modifications importantes de leurs caractéristiques : baisse de la teneur en carbone organique et du rapport $\mathrm{C} / \mathrm{N}$, augmentation $\mathrm{du} \mathrm{pH}$ et baisse du phosphore extractible, réduction notable des polysaccharides et accroissement des composés analogues aux substances humiques.

La consommation d'oxygène d'échantillons de composts seuls et la production de gaz carbonique par des mélanges sol + composts est fonction de l'origine des produits et surtout de l'âge du compost reflétant le degré de stabilisation atteint par les matières organiques.
\end{abstract}

Mots clés additionnels : Amendement organique, compost, valorisation des déchets, dégradation biotique, respirométrie, composés humiques, polysaccharides, technologie de compostage.

An analytical study was made on samples of urban compost and ground urban waste from 14 treatment plants and landfilling sites in France. Samples were taken at different stages of waste processing and various physical and chemical parameters determined. Following characterization of the organic matter fractions, respirometric experiments were carried out. Fresh urban wastes showed great variation according to their origin and the analysed parameters were then greatly modified during composting or in sanitary landfills : decrease in organic carbon content and $\mathrm{C} / \mathrm{N}$ ratio, increased $\mathrm{pH}$ and reduction of available phosphorus. Polysaccharide content was reduced by composting while humic-like substances increased. The oxygen consumption of composts alone and the carbon dioxide evolved from soil-compost mixtures depended of the origin of the waste and especially on the age of the compost, serving as an indicator of the degree of stabilization of the wastes.

Additional key words : Soil amendments, waste disposal, microbial degradation, respirometry, humic substances, polysaccharides, compost technology.

\section{INTRODUCTION}

La valorisation agricole des composts urbains est une réponse intéressante aux problèmes du recyclage des déchets (BASALO, 1974; SALAUN, 1976; GOlueKe, 1977, 1979; CHAMBOLle, 1978) et à celui du maintien du taux de matières organiques dans certains sols (TIETJEN, 1975). Le compost urbain qui résulte de la transformation biologique des ordures ménagères (POMMEL \& BERNARD, 1975 ; BASAlO, 1978 ) peut présenter un intérêt agronomique (ANSTETT, 1978) en tant que fertilisant ou source de matière organique (VLAMIS \& WILLIAMS, 1972).

Le compostage modifie profondément les caractéristiques analytiques des ordures ménagères en provoquant une transformation des produits carbonés (INOKO et al., 1979) avec des pertes importantes de gaz carbonique. Cette évolution très dépendante $d u$ 
traitement technologique conduit à un produit organique d'une stabilité biologique accrue et peut être alors considéré comme mûr.

L'objectif de nos recherches, entreprises sous l'égide du Ministère de l'Environnement, a consisté à :

- suivre l'évolution des caractéristiques physicochimiques des ordures ménagères au cours du compostage,

- caractériser les formes de carbone organique contenues dans les ordures ménagères et étudier l'évolution respective de ces différentes formes au cours du traitement,

- enregistrer enfin l'activité biologique des composts, avant ou après incorporation dans le sol, au moyen de mesures respirométriques qui constituent une approche globale pour suivre l'évolution de la matière organique de ces produits (CHROMETZKA, 1968 ; ALLENSPACH, 1969 ; BENISTANT, 1978 ; NICOLARDOT, 1979).

Afin d'atteindre ces objectifs, nous avons été amenés à travailler sur un large échantillonnage de produits qui englobe les filières de traitement les plus couramment utilisées. Le choix de ces matériaux d'étude a pu être effectué grâce à l'expérience acquise par différents auteurs (BERNARD, 1977 ; JUSTE \& SOLDA, 1977 ; COLIN, 1978). Enfin, nous avons recherché à enregistrer l'impact de la technologie de traitement des ordures sur la modification de leur évolution en comparant différentes filières de traitement (fermentation contrôlée, fermentation sur aire, décharge d'ordures broyées).

\section{MATÉRIELS ET MÉTHODES}

\section{A. Matériel d'étude}

\section{Origine des échantillons}

Cette étude a porté sur un ensemble d'échantillons de composts ou ordures broyées provenant de 14 usines de compostage ou décharges situées en France (tabl. 1). Dans chacune des usines, les ordures ménagères font l'objet d'un certain nombre de prétraitements (tri, broyage, criblage) qui visent à homogénéiser, affiner, humidifier le produit destiné à la fermentation. Les ordures sont ensuite acheminées vers les dispositifs de fermentation. A ce niveau, les fabricants distinguent 3 grands types de traitements :

- le compostage contrôlé : les ordures passent par un hygiénisateur ou fermenteur pendant 3 à $15 \mathrm{j}$ selon les usines, avant d'être mises en tas pour une durée variable pouvant être supérieure à 1 an ;

- la fermentation sur aire : les ordures sont mises en tas immédiatement après les opérations de prétraitement avec retournement périodique des tas ;

- la mise en décharge : après le seul broyage, les ordures sont déposées dans une décharge contrôlée pour une durée généralement plus longue que celle requise par le compostage sur aire. Elles peuvent être

TABLEAU

Tableau récapitulatif de l'origine des composts et des essais mis en place. Origin of the composts and outline of the experiments.

\begin{tabular}{|c|c|c|c|c|c|}
\hline $\begin{array}{c}\text { Type de traitement } \\
\text { des ordures ménagères }\end{array}$ & $\begin{array}{c}\text { Usine } \\
\text { (constructeur) }\end{array}$ & Localité & $\begin{array}{l}\text { Code } \\
\text { compost }\end{array}$ & $\begin{array}{l}\text { Particularités des } \\
\text { essais réalisés }\end{array}$ & $\begin{array}{c}\text { Age des différents } \\
\text { échantillons étudiés (jours) }\end{array}$ \\
\hline $\begin{array}{l}\text { Fermentation } \\
\text { sur aire }\end{array}$ & $\begin{array}{l}\text { A (PRAT) } \\
\text { B (O.A.D.) }\end{array}$ & $\begin{array}{l}\text { Châlons/Saône } \\
\text { La Loupe }\end{array}$ & $\begin{array}{l}\mathrm{Aa} \\
\mathrm{Ab}^{*} \\
\mathrm{Ac}^{*} \\
\mathrm{~B}\end{array}$ & $\begin{array}{l}\text { Ordures ménagères } \\
\text { Ordures ménagères } \\
\text { Ordures ménagères } \\
\text { Ordures ménagères }\end{array}$ & $\begin{array}{l}4-60-120-180-240 \\
4-60-120-240 \\
8-21-120-150-210 \\
0-60-120-240\end{array}$ \\
\hline $\begin{array}{l}\text { Fermentation } \\
\text { contrôlée }\end{array}$ & $\begin{array}{l}\text { D (CAREL-FOUCHE- } \\
\text { LANGUEPIN) } \\
\text { E (CAREL-FOUCHE- } \\
\text { LANGUEPIN) } \\
\text { 2) Cylindre fermenteur } \\
\text { F (DANO) } \\
\text { G (DANO) }\end{array}$ & $\begin{array}{l}\text { Montbéliard } \\
\text { Orléans } \\
\text { Pontenx-les-Forges } \\
\text { Tournan-en-Brie }\end{array}$ & $\begin{array}{l}\mathrm{Ca} \\
\mathrm{Cb} \\
\mathrm{Cc} \\
\mathrm{Cd} \\
\mathrm{Ce} \\
\mathrm{Cf} \\
\mathrm{Da} \\
\mathrm{E}\end{array}$ & $\begin{array}{l}\text { Mélange ordures ménagères et } \\
\text { - boues digérées anaérobies } \\
\text { - boues fraîches } \\
\text { - boues digérées aérobies } \\
\text { - matières de vidange } \\
\text { - lisier } \\
\text { Ordures ménagères seules } \\
\text { Ordures ménagères } \\
\text { Ordures ménagères } \\
\text { Ordures ménagères } \\
\text { Ordures ménagères } \\
\text { Ordures ménagères }\end{array}$ & $\begin{array}{l}6-60-120-240 \\
6-60-120-240 \\
6-60-120-240 \\
6-60-120-240 \\
6-60-120-240 \\
0-60-120-187-210 \\
6-60-120-180 \\
0-30-60-90-120-150-180- \\
210-240 \\
4-60-120 \\
4-60-120-240 \\
4-60-120\end{array}$ \\
\hline $\begin{array}{l}\text { Ordures broyées } \\
\text { mises en décharge }\end{array}$ & $\begin{array}{l}\text { Broyeur à marteaux } \\
\text { H } \\
\text { I } \\
\text { J } \\
\text { K } \\
\text { L } \\
\text { M } \\
\text { N }\end{array}$ & $\begin{array}{l}\text { Chateaubriant } \\
\text { Auxerre } \\
\text { Digne } \\
\text { Manosque } \\
\text { Barcelonnette } \\
\text { Lons-le-Saunier } \\
\text { Bar/Aube }\end{array}$ & $\begin{array}{l}\mathrm{H} \\
\mathrm{I} \\
\mathrm{J}^{*} \\
\mathrm{~K}^{*} \\
\mathrm{~L}^{*} \\
\mathrm{M}^{*} \\
\mathrm{~N}^{*}\end{array}$ & $\begin{array}{l}\text { Ordures ménagères } \\
\text { Ordures ménagères } \\
\text { Ordures ménagères } \\
\text { Ordures ménagères } \\
\text { Ordures ménagères } \\
\text { Ordures ménagères } \\
\text { Ordures ménagères }\end{array}$ & $\begin{array}{l}0-240-720 \\
0-60-120-240 \\
15-720-1800 \\
60-180-270 \\
105-540-1260 \\
15-60-105-165-360 \\
15-45-90-105\end{array}$ \\
\hline
\end{tabular}

* Echantillons conservés à $4{ }^{\circ} \mathrm{C}$ dans leur état d'humidité initial. 
reprises par la suite, criblées et utilisées à des fins agricoles.

\section{Prélèvements}

Les échantillons sont soit prélevés à l'occasion du retournement des tas, soit prélevés à différents niveaux du tas après ouverture d'une saignée verticale de manière à obtenir un échantillon aussi représentatif que possible $(10$ à $15 \mathrm{~kg})$.

\section{Préparation des échantillons}

Les quantités de produit utilisées au laboratoire étant très faibles, il est indispensable d'en réduire fortement l'hétérogénéité. Dans ce but, les échantillons prélevés en usine sont broyés (broyeur à marteaux ou appareil de type "mixer ") après séchage (à environ $45^{\circ} \mathrm{C}$ ) pendant 5 à $6 \mathrm{j}$ et élimination des matériaux non broyables (métaux, matières plastiques dures...). Le produit est alors conservé à $+4{ }^{\circ} \mathrm{C}$ sans risque d'évolution notable.

\section{B. Méthodes d'analyse}

L'humidité est obtenue par séchage à $105^{\circ} \mathrm{C}$ pendant $24 \mathrm{~h}$ et l'humidité équivalente déterminée par la méthode de BOUYOUCOS (1929) adaptée. Le pH est mesuré sur une suspension de compost dans l'eau (rapport 1/5).

Le carbone organique total (C.O.T.) est dosé soit par voie humide (ANNE, 1945), soit par voie sèche à l'aide d'un appareil de type carmhograph 12 de WosTHOFF après destruction préalable des carbonates par $\mathrm{HCl} 4 \mathrm{~N}$. Dans ce dernier cas, on mesure la totalité du carbone organique y compris celui des matières plastiques.

L'azote organique total dont on soustrait l'azote minéral extrait par $\mathrm{KCl} \mathrm{N}$ (rapport compost/solution $=1 / 20$ ) est dosé par la méthode Kjeldahl (BREMNER, 1965). Le dosage des ions ammonium et nitrate est réalisé par distillation (BREMNER, 1965).

Les éléments totaux sont dosés par absorption atomique $(\mathrm{K}, \mathrm{Ca}, \mathrm{Mg}, \mathrm{Na})$ et colorimétrie $(\mathrm{P})$ après calcination à $550^{\circ} \mathrm{C}$ et reprise des cendres par $\mathrm{HCl}$.

Le phosphore extractible est obtenu par la méthode JORET-HÉBERT (1955), le choix de cette méthode étant guidé par le pH élevé des composts.

Afin de déterminer la part de la fraction glucidique dans la matière organique de divers composts, nous avons procédé au dosage des glucides hydrosolubles et des glucides hydrolysables. La méthode utilisée est celle de BRINK et al. (1960) pour les sols, modifiée par GUCKERT (1973). Elle utilise 2 extractions à $80^{\circ} \mathrm{C}$ :
- la $1^{\text {re }}$, à l'eau distillée, extrait les sucres libres, - la $2^{\mathrm{e}}$ consiste en une hydrolyse par l'acide sulfurique $3 \mathrm{~N}$ et permet d'extraire les sucres des polysaccharides. Le dosage est réalisé par colorimétrie après réaction avec le phénol selon la méthode de DuBoIs et al. (1956).

Pour suivre l'humification des matières organiques présentes dans les composts, nous avons fait appel à des techniques d'extraction dérivées de celles utilisées pour l'étude de la matière organique des sols (DUCHAUFOUR \& JACQUiN, 1966). Le compost est traité à l'aide d'un réactif alcalin $(\mathrm{NaOH} 0,1 \mathrm{~N})$. L'extrait obtenu est séparé en 2 fractions par abaissement du $\mathrm{pH}$ à la valeur 1 : les acides humiques (AH) qui floculent lors de l'acidification et les acides fulviques (AF) restés en solution. Le dosage du carbone contenu dans les fractions est effectué par voie sèche au carmhograph de WOSTHOFF. Les résultats sont exprimés sous la forme de 2 rapports :

Taux d'extraction à la soude $\mathrm{N} / 10$

$$
\frac{\text { C extrait par NaOH N/10 }}{\text { C.O.T. }} \text { (en p. 100). }
$$

Taux de carbone acido-soluble

$$
\frac{\mathrm{C} \text { extrait par } \mathrm{NaOH} \mathrm{N} / 10 \text { soluble à } \mathrm{pH} 1}{\mathrm{C} \text { extrait par } \mathrm{NaOH} \mathrm{N} / 10}
$$

\section{Etudes respirométriques}

\section{Respirométrie électrolytique de composts seuls}

Les mesures respirométriques sont réalisées à l'aide de respiromètres électrolytiques mis au point par GERMON \& VERGUET (1974). Ces appareils permettent d'enregistrer la cinétique de consommation d'oxygène sur des temps relativement longs. Après avoir réalisé des mesures pendant $28 \mathrm{j}$ sur les composts de l'usine $\mathrm{A}$, les $1^{\text {ers }}$ résultats ont permis de réduire à $7 \mathrm{j}$ la durée d'incubation pour les échantillons traités ultérieurement $(C, D, E)$. Les mesures respirométriques sont effectuées à $20^{\circ} \mathrm{C}$ avec un minimum de 4 répétitions. Les incubations sont réalisées sur des échantillons de $10 \mathrm{~g}$ de compost (poids sec) humidifiés aux $2 / 3 \mathrm{de}$ leur humidité équivalente, le piégeage du gaz carbonique étant assuré par $30 \mathrm{ml}$ de soude $2 \mathrm{~N}$.

\section{Respirométrie de mélanges sol + compost}

L'étude de la minéralisation de 73 composts d'origine et d'âge différents dans un horizon Ap d'un sol brun lessivé (tabl. 2) est réalisée en utilisant un dispositif respirométrique d'incubation conçu par GUCKERT

TABLEAU 2

\begin{tabular}{|c|c|c|c|c|c|c|c|c|c|c|c|c|c|}
\hline \multicolumn{6}{|c|}{ Granulométrie } & \multicolumn{8}{|c|}{ Complexe absorbant } \\
\hline $\mathrm{A}$ & $\mathrm{LF}$ & LG & $\mathrm{SF}$ & $\mathrm{SG}$ & \multirow{2}{*}{$\begin{array}{c}\text { Capacité } \\
\text { au champ } \\
\% \mathbf{0}\end{array}$} & $\mathrm{C}$ & $\mathrm{N}$ & $\mathrm{C} / \mathrm{N}$ & $\mathrm{Ca}^{2+}$ & $\mathrm{Mg}^{2+}$ & $\mathrm{K}^{+}$ & $\mathrm{T}$ & \multirow{2}{*}{$\begin{array}{c}\mathrm{P}_{2} \mathrm{O}_{5} \\
\%\end{array}$} \\
\hline & & $\%$ & & & & $\%$ & $\%$ & & \multicolumn{4}{|c|}{$\mathrm{meq} / 100 \mathrm{~g}$} & \\
\hline 17,9 & 22,1 & 15,0 & 31,3 & 13,6 & 28,0 & 1,57 & 0,17 & 9,3 & 14,5 & 1,1 & 0,7 & 12,7 & 0,40 \\
\hline
\end{tabular}

Analyse physico-chimique du sol utilisé.

Physico-chemical analysis of soil. 
et al. (1968). Il permet d'apprécier la minéralisation dans un sol d'un matériau organique quelconque introduit par le dosage périodique du $\mathrm{CO}_{2}$ dégagé.

Des mélanges sol + compost ( 3 répétitions) sont préparés en ajoutant à $100 \mathrm{~g}$ de sol une quantité de compost équivalente à $0,235 \mathrm{~g}$ de carbone organique, apport correspondant à environ $30 \mathrm{t}$ de compost sec par ha pour un produit contenant 20 p. 100 de carbone. L'humidité du sol a été ajustée à $80 \mathrm{p} .100$ de la capacité au champ. Le $\mathrm{CO}_{2}$ dégagé est piégé dans de la soude $2 \mathrm{~N}$ et dosé par titrimétrie après précipitation de $\mathrm{Ba}\left(\mathrm{CO}_{3}\right)_{2}$. Les mesures sont réalisées chaque jour, pendant la $1^{\text {re }}$ décade puis tous les $2 \mathrm{j}$ jusqu'au terme de l'expérience effectuée à $28^{\circ} \mathrm{C}$. Les résultats sont exprimés en pourcentage cumulé de carbone minéralisé sous forme $\mathrm{CO}_{2}$ par rapport au carbone initial total du mélange. Ce mode d'expression appelé Taux de Minéralisation Globale du mélange (T.M.G.), permet de rendre compte de l'activité minéralisatrice du sol en présence de divers composts. La quantité de matière organique des composts minéralisée pendant l'expérience est calculée à partir du rapport suivant :

$$
=\% \frac{\mathrm{C} \text { dégagé }(\text { sol }+ \text { compost })-\mathrm{C} \text { dégagé sol témoin }}{\mathrm{C} \text { introduit dans le sol }} .
$$

Ce mode d'expression ne permet évidemment pas de tenir compte des éventuels "priming effects » dus à l'introduction des composts dans le sol et ne constituera donc qu'une estimation imparfaite de la dégradation réelle de la matière organique des composts.

\section{RÉSULTATS ET DISCUSSION}

\section{A. Evolution des caractéristiques physico-chimiques}

\section{Matériaux de départ}

L'ensemble des résultats est présenté dans le tableau 3 : le carbone organique total correspond sensiblement à 28 p. 100 de la matière sèche des composts analysés. Etant donnée la composition très hétérogène de ces matériaux et la diversité des provenances, on peut considérer que les variations observées sont modérées.

La teneur moyenne en azote total est de 1 p. 100 avec des valeurs extrêmes de 0,67 et 1,4 p. 100 .
L'amplitude de variation de l'azote total est à rattacher à l'addition de matériaux plus ou moins riches en azote (boues d'épuration, matières de vidange, lisiers...) aux ordures ménagères. Il en résulte un $\mathrm{C} / \mathrm{N}$ moyen des ordures ménagères de 30 avec des fluctuations de 25 à 40 , les valeurs les plus basses étant observées pour les ordures enrichies de boues d'épuration. On note que l'essentiel de l'azote se trouve sous forme organique et que les matériaux de départ sont relativement pauvres en azote minéral; la teneur en phosphore total par contre, n'est pas négligeable : elle représente près de $2 \mathrm{p} .100$ de la matière sèche dont 20 p. 100 est extractible à l'oxalate d'ammonium.

Certains cations sont également présents en quantités importantes. Leur concentration totale permet le classement suivant par ordre décroissant : $\mathrm{Ca}, \mathrm{Na}, \mathrm{K}$, $\mathrm{Mg}$. Le pH est voisin de la neutralité. L'humidité équivalente, de valeur élevée, laisse déjà entrevoir l'intérêt de ces matériaux sur les propriétés physiques du sol ou lors de leur utilisation comme substrat de culture.

En définitive, les ordures ménagères en raison de leur rapport $\mathrm{C} / \mathrm{N}$ élevé et de leur richesse en matériaux fermentescibles constituent une matière organique susceptible d'évoluer biologiquement. C'est pourquoi leur éventuelle utilisation en agriculture nécessite soit une transformation préalable par compostage, soit des modalités d'apport et d'enfouissement directement au champ favorisant une évolution et une stabilisation rapides sans préjudice pour les cultures.

\section{Evolution des ordures ménagères au cours du compostage}

\section{a) Cas de l'usine $A$}

L'échantillonnage bi-mensuel d'un même tas de compost de l'usine A montre (tabl. 4) une nette évolution des paramètres physiques et chimiques au cours du compostage, notamment une baisse importante du taux de carbone organique due à la minéralisation. La teneur en azote total présente une augmentation sensible au cours de la fermentation : cette variation correspond en fait à un enrichissement relatif en azote de la matière sèche résiduelle du compost, la quantité totale d'azote allant en fait en diminuant comme l'illustre la figure 2 . Les teneurs en azote minéral sont toujours faibles (environ 3 p. 100 de l'azote total) et leur évolution est typique de ce que l'on rencontre

TABLEAU 3

Caractérisation chimique des produits de départ (1)

(résultats moyens obtenus à partir des différentes stations de traitement des ordures étudiées exprimés par rapport à la matière sèche)

Chemical characteristics of initial refuse

(mean values obtained from the different composting plants expressed as \% dry matter)

\begin{tabular}{|c|c|c|c|c|c|c|c|c|c|c|c|c|c|}
\hline & \multirow{2}{*}{$\begin{array}{c}\text { Carbone } \\
\text { organique } \\
\text { total } \\
\%\end{array}$} & \multirow[b]{2}{*}{$\begin{array}{c}\text { Azote } \\
\text { total } \\
\%\end{array}$} & \multirow[b]{2}{*}{$\mathrm{C} / \mathrm{N}$} & \multicolumn{2}{|c|}{ Azote minéral } & \multicolumn{2}{|c|}{ Phosphore } & \multirow{2}{*}{$\begin{array}{l}\text { Potassium } \\
\qquad \begin{array}{l}\text { total } \\
\mathrm{K} \% \mathrm{oo}\end{array}\end{array}$} & \multirow{2}{*}{$\begin{array}{l}\text { Calcium } \\
\text { total } \\
\mathrm{Ca} \%\end{array}$} & \multirow{2}{*}{$\begin{array}{c}\text { Magnésium } \\
\text { total } \\
\mathrm{Mg} \%\end{array}$} & \multirow{2}{*}{$\begin{array}{l}\text { Sodium } \\
\begin{array}{c}\text { total } \\
\mathrm{Na} \%\end{array}\end{array}$} & \multirow{2}{*}{$\begin{array}{c}\text { Humidité } \\
\text { équivalente } \\
\%_{0}\end{array}$} & \multirow[b]{2}{*}{$\mathrm{pH}$ eau } \\
\hline & & & & $\begin{array}{l}\mathrm{N}-\mathrm{NH}_{4}^{+} \\
\mathrm{N} \% \text { oo }\end{array}$ & $\begin{array}{l}\mathrm{N}-\mathrm{NO}_{3}^{-} \\
\mathrm{N} \% \mathrm{O}\end{array}$ & $\begin{array}{c}\text { total } \\
\mathrm{P} \%\end{array}$ & $\begin{array}{l}\text { JORET- } \\
\text { HÉBERT } \\
\mathrm{P}^{\%} \% \text { oO }\end{array}$ & & & & & & \\
\hline \multirow{3}{*}{$\begin{array}{l}\text { Valeurs moyennes } \\
\text { Valeurs extrêmes }\end{array}$} & 27,6 & 0,95 & 29,9 & 0,20 & 0,02 & 2,1 & 0,45 & 4,8 & 37,5 & 3,2 & 21,6 & 101,0 & 7,1 \\
\hline & 18,3 & 0,67 & 23,9 & 0.10 & 0,06 & 1.4 & 0,38 & 3,2 & 25,4 & 1,3 & 2,8 & 68,3 & 6,2 \\
\hline & 33,7 & 1,40 & 44,9 & 0.30 & 0.04 & 2.8 & 0.53 & 8,4 & 59,5 & 6,5 & 45,6 & 152,5 & 7.9 \\
\hline $\begin{array}{l}\text { Coefficient de variation } \% \\
\text { Nombre de types de composts } \\
\text { analysés }\end{array}$ & 12,9 & 18,0 & 19,6 & 40,3 & 66,8 & 11,5 & 24,8 & $\$ 2,1$ & 38,0 & 61,4 & 87,1 & 37,3 & - \\
\hline
\end{tabular}

(1) Par produit de départ, on entend les ordurcs ménagères qui vont être mises en fermentation sur aire ou décharge et qui ont dans certains cas subi les diverses opérations de prétraitement et préfermentation. 
TABLEAU 4

Evolution des caractéristiques physico-chimiques du compost de l'usine A (échantillons Aa) exprimées par rapport à la matière sèche. Physical and chemical characteristics of the compost from composting plant A (samples Aa) expressed as \% dry matter.

\begin{tabular}{|c|c|c|c|c|c|c|}
\hline \multirow{2}{*}{ Analyses } & & \multicolumn{5}{|c|}{ Age du compost en jours } \\
\hline & & 4 & 60 & 120 & 180 & 240 \\
\hline Humidité équivalente & $\%$ & 152,5 & 147,3 & 132,0 & 53,0 & 49,0 \\
\hline Perte au feu à $1110^{\circ} \mathrm{C}$ & $\%$ & 69,2 & 61,0 & 49,6 & 39,0 & 47,9 \\
\hline Carbone organique (méth. ANNE) & $\%$ & 33,7 & 30,4 & 23,7 & 16,8 & 14,2 \\
\hline Azote Kjeldahl & $\%$ & 0,98 & 0,96 & 1,19 & 1,10 & 1,19 \\
\hline Azote minéral N-NH${ }_{4}^{+}$ & $\%$ & 0,29 & 0,14 & 0,04 & 0,06 & 0,03 \\
\hline $\mathrm{N}-\mathrm{NO}_{3}^{-\frac{4}{3}}$ & $\%$ & 0,00 & 0,00 & 0,00 & 0,12 & 0,30 \\
\hline $\mathrm{C} / \mathrm{N}$ & & 34,4 & 31,7 & 19,9 & 15,3 & 12,0 \\
\hline pH eau & & 7,1 & 7,6 & 8,0 & 8,0 & 8,0 \\
\hline P JORET-HÉBERT & $\mathrm{d} / \mathrm{oo}$ & 0,49 & 0,40 & 0,34 & 0,27 & 0,29 \\
\hline
\end{tabular}

dans les différents tas de composts : faible niveau d'azote ammoniacal et traces de nitrate en début de compostage, puis formation de nitrate en fin de maturation. Cette maturation se traduit par un abaissement du rapport $\mathrm{C} / \mathrm{N}$, qui passe de 34 à 12 , indiquant une stabilisation des composés organiques. De même l'humidité équivalente du produit chute, et le taux de phosphore JORET-HÉBERT présente une diminution nette, ce dernier point étant à mettre en relation avec l'élévation concomitante du $\mathrm{pH}$.

\section{b) Comparaison de l'évolution des ordures dans 3 procédés de traitement}

Les figures 1 et 2 illustrent l'évolution du carbone organique et de l'azote organique exprimés respectivement en p. $100 \mathrm{du}$ carbone organique initial et de l'azote organique initial, la fraction minérale étant considérée comme restant constante au cours du processus évolutif. En fermentation contrôlée (usine E) où les ordures ménagères subissent une préfermentation suivie d'une évolution en tas sur aire avec 2 réhumidifications et des retournements mensuels, la décroissance du carbone organique et de l'azote total

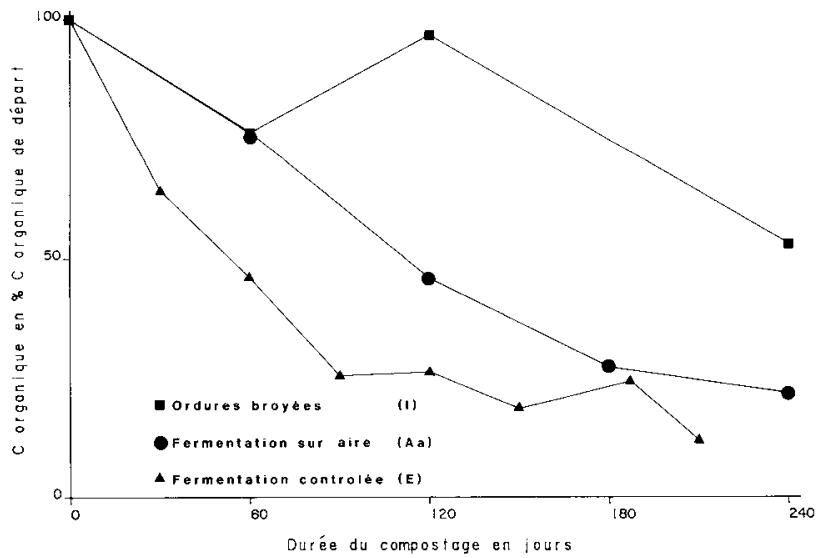

Figure 1

Evolution de la quantité de carbone organique dans 3 types de traitement des ordures ménagères : les taux de carbone aux différentes dates sont rapportés à la quantité initiale de carbone, la quantité de cendres ayant été considérée constante durant cette période.

Change in organic carbon content during waste composting in three types of treatment plants : carbon levels at different sampling dates are related to the initial quantity of carbon. Otherwise ash content has been considered to be constant.

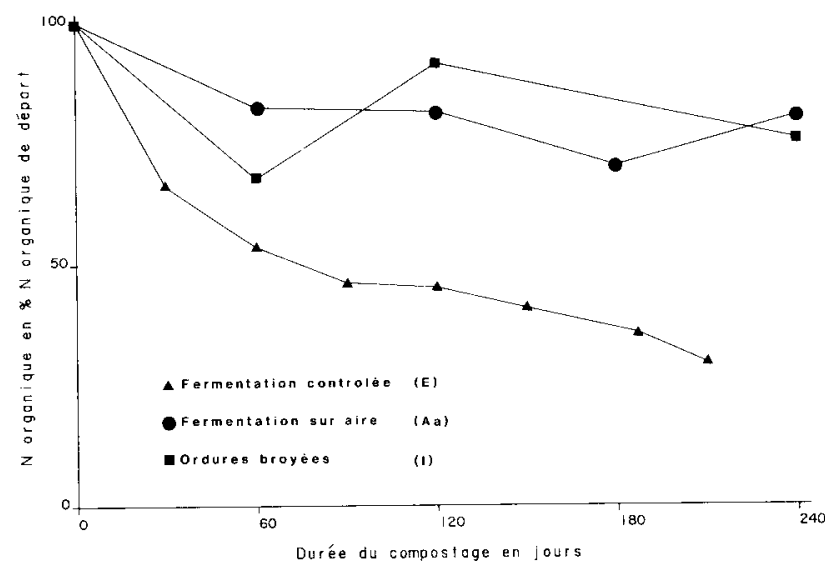

Figure 2

Evolution de la quantité d'azote total dans 3 types de traitement des ordures ménagères : les taux d'azote aux différentes dates sont rapportés à la quantité initiale d'azote, la quantité de cendres ayant été considérée constante durant cette période.

Change in total nitrogen content during waste composting in three types of treatment plants: nitrogen levels at different sampling dates are related to the initial quantity of nitrogen. Otherwise ash content has been considered to be constant.

apparaissent comme étant les plus rapides. Pour l'azote, ces pertes se font vraisemblablement par volatilisation d'ammoniac, grâce à l'action combinée de l'aération, de la température et de l'alcalinisation du compost. Pour la fermentation directe sur aire (usine A), l'évolution est plus lente. En ce qui concerne les ordures broyées (usine I), la dégradation paraît $a$ priori encore plus lente, mais elle est en partie masquée par une hétérogénéité très grande des échantillons.

\section{Caractéristiques analytiques moyennes des composts après fermentation}

Les valeurs moyennes des différentes caractéristiques analytiques des composts présentées ici (tabl. 5) sont calculées à partir des résultats obtenus sur les usines étudiées après une période de compostage de $240 \mathrm{j}$.

Les valeurs du $\mathrm{pH}$ ont tendance à s'accroître au cours du compostage pour atteindre des chiffres moyens voisins de 8 .

Pour tous les procédés, on observe une diminution 


\section{TABLEAU 5}

Tableau récapitulatif des valeurs moyennes de quelques caractéristiques des ordures fraîches et des ordures compostées pendant 240 jours exprimées par rapport à la matière sèche.

Mean values of characteristics of fresh wastes and 240-day old compost, expressed as \% dry matter.

\begin{tabular}{|c|c|c|c|c|c|c|c|c|}
\hline & $\begin{array}{c}\text { Carbone } \\
\text { organique } \\
\text { total } \\
\%\end{array}$ & $\begin{array}{c}\text { Azote } \\
\text { total } \\
\\
\% 0\end{array}$ & $\mathrm{C} / \mathrm{N}$ & $\begin{array}{r}\mathrm{A} \\
\mathrm{mi} \\
\mathrm{N}-\mathrm{NH} \\
\% \text { io }\end{array}$ & $\begin{array}{l}\mathrm{e} \\
\mathrm{al} \\
-\mathrm{NO}_{3}^{-} \\
\quad \%\end{array}$ & $\begin{array}{c}\text { Phosphore } \\
\text { JORET- } \\
\text { HÉBERT } \\
\% \text { oo }\end{array}$ & $\begin{array}{c}\text { Humidité } \\
\text { équivalente } \\
\%\end{array}$ & $\mathrm{pH}$ \\
\hline Valeurs moyennes & 14,2 & 0,95 & 15,0 & 0,03 & 0,14 & 0,18 & 45,8 & 7,7 \\
\hline Valeurs extrêmes & $6,4-20,2$ & $0,5-1,35$ & - & $0,03-0,27$ & $0,04-0,30$ & $0,14-0,29$ & $40,6-50,1$ & $7,2-8,1$ \\
\hline $\begin{array}{l}\text { Nombre de types de } \\
\text { compost analysés }\end{array}$ & 15 & 15 & - & 5 & 5 & 5 & 4 & 15 \\
\hline
\end{tabular}

régulière de la teneur en eau à $\mathrm{pF} 2,5$ consécutive à la perte de matière organique ; 8 mois de compostage conduisent à une réduction de moitié de la capacité de rétention en eau (inférieure en moyenne à 50 p. 100), ce qui entraîne une diminution de l'intérêt agronomique de ces composts en ce qui concerne leur qualité au niveau de la rétention en eau.

La teneur en carbone organique initiale moyenne des ordures ménagères, de l'ordre de $30 \mathrm{p} .100$, décroît de moitié entre 6 et $240 \mathrm{j}$ de compostage. Si la concentration en azote total paraît augmenter en valeur moyenne, le compostage s'accompagne néanmoins de pertes de composés azotés comme cela a été montré précédemment. Finalement, après $240 \mathrm{j}$ de fermentation, la teneur en azote total est voisine de 1 p. 100 . Le rapport $\mathrm{C} / \mathrm{N}$, généralement élevé dans le matériel de départ, décroît régulièrement dans le temps pour aboutir à une valeur moyenne de l'ordre de 15 ; cette diminution correspond à une évolution des matières organiques vers des formes plus stables et plus humifiées.
Les teneurs en azote minéral des composts étudiés sont relativement faibles. Au cours du temps, on observe une diminution marquée de $\mathrm{N}-\mathrm{NH}_{4}^{+}$s'accompagnant d'une libération d'azote nitrique en fin de compostage. Précisons cependant que les valeurs fluctuent considérablement d'une usine à l'autre. Les teneurs moyennes en phosphore JORET-HÉBERT diminuent régulièrement en fonction du temps. Après $240 \mathrm{j}$ les teneurs sont voisines de $0,2 \mathrm{p} .1000$.

\section{B. Evolution de différentes fractions carbonées des composts}

Une fraction du carbone organique des ordures ménagères avant compostage sur aire est sous forme glucidique (tabl. 6). Le carbone des sucres solubles représente environ 2 p. $100 \mathrm{du}$ carbone organique total, les sucres hydrolysables étant plus abondants avec 16 p. 100 , dépassant même 40 p. 100 dans certains cas.

La teneur en composés glucidiques chute considéra-

TABLEAU 6

Evolution moyenne des différentes formes de carbone organique au cours du compostage.

Change in the proportions of the different forms of organic matter during composting.

\begin{tabular}{|c|c|c|c|c|c|}
\hline & & \multicolumn{4}{|c|}{ Age du compost (jours) } \\
\hline & & 0 à 6 & 60 & 120 & 240 \\
\hline \multirow{4}{*}{ (\%oo M.S.) hydrosolubles } & $\begin{array}{r}\text { Valeurs } \\
\text { moyennes }\end{array}$ & 12,3 & 6,2 & 4,5 & 3,9 \\
\hline & $\begin{array}{r}\text { Valeurs } \\
\text { extrêmes }\end{array}$ & $\begin{array}{c}3,9-22,1 \\
(18)\end{array}$ & $\begin{array}{l}2,4-10,0 \\
(14)\end{array}$ & $\begin{array}{c}0,8-7,4 \\
(14)\end{array}$ & $\begin{array}{c}1,2-8,8 \\
(14)\end{array}$ \\
\hline & $\begin{array}{r}\text { Valeurs } \\
\text { moyennes }\end{array}$ & 99,0 & 72,1 & 53,2 & 39,4 \\
\hline & $\begin{array}{r}\text { Valeurs } \\
\text { extrêmes }\end{array}$ & $\begin{array}{c}42,5-169,6 \\
(18)\end{array}$ & $\begin{array}{c}35,2-129,2 \\
(14)\end{array}$ & $\begin{array}{c}30,8-102,1 \\
(14)\end{array}$ & $\begin{array}{c}20,4-78,3 \\
\text { (14) }\end{array}$ \\
\hline $\mathrm{C}$ extrait à $\mathrm{NaOH} \%$ & $\begin{array}{r}\text { Valeurs } \\
\text { moyennes }\end{array}$ & 7,6 & 7,9 & 9,8 & 11,8 \\
\hline C.O.T. & $\begin{array}{r}\text { Valeurs } \\
\text { extrêmes }\end{array}$ & $\begin{array}{c}4,1-13,1 \\
\text { (13) }\end{array}$ & $\begin{array}{l}5,2-10,2 \\
\text { (14) }\end{array}$ & $\begin{array}{c}5,3-15,7 \\
(14)\end{array}$ & $\begin{array}{c}6,0-19,3 \\
(14)\end{array}$ \\
\hline $\mathrm{C}$ extrait soluble à $\mathrm{pH} 1$ & $\begin{array}{r}\text { Valeurs } \\
\text { moyennes }\end{array}$ & 0,86 & 0,82 & 0,77 & 0,64 \\
\hline C extrait & $\begin{array}{r}\text { Valeurs } \\
\text { extrêmes }\end{array}$ & $\begin{array}{c}0,80-0,94 \\
(12)\end{array}$ & $\begin{array}{c}0,73-0,95 \\
(13)\end{array}$ & $\begin{array}{c}0,71-0,85 \\
(13)\end{array}$ & $\begin{array}{c}0,35-0,90 \\
\text { (13) }\end{array}$ \\
\hline
\end{tabular}

(Nombre de types de composts analysés); (number of composts analysed). 
blement au cours du compostage. Elle ne représente plus que 4 à 10 p. 100 environ de la matière organique après $240 \mathrm{j}$. La quantité de polysaccharides extractibles après hydrolyse acide présente en fin de compostage pourrait correspondre pour partie à des composés de néosynthèse microbienne. Ces données sont en accord avec celles d'INOKO et al. (1982) qui indiquent une réduction des teneurs en hémicellulose et cellulose au cours du compostage.

Les matières organiques des composts urbains sont peu solubles dans les réactifs alcalins et le taux d'extraction par la soude augmente en cours de compostage. Cependant, cette évolution est plutôt liée à la diminution du stock de carbone organique qu'à l'augmentation des matières organiques extractibles à la soude.

Le fractionnement de la solution d'extraction (floculation par acidification à $\mathrm{pH}$ 1) permet de rendre compte de l'état de polymérisation des composés organiques extractibles. Le taux de carbone acidosoluble (correspondant aux acides fulviques) diminue significativement durant le compostage, indiquant la polycondensation des substances organiques du compost au cours du temps. Ces résultats sont conformes à ceux de SUGAHARA \& INOKO (1981) qui rapportent une augmentation des composés humiques par rapport aux acides fulviques au cours de la maturation.

\section{Etudes respirométriques}

\section{Respirométrie électrolytique de composts évoluant seuls}

La figure 3 présente les consommations cumulées d'oxygène en $28 \mathrm{j}$ pour les composts provenant de l'usine A. Les courbes montrent que l'activité respiratoire diminue au fur et à mesure que vieillit le compost; on constate également que ces observations peuvent être effectuées dès le début de l'incubation. A titre d'exemple, la figure 4 illustre les consommations cumulées des produits de différents âges ayant pour origine l'usine $\mathrm{C}$ lors de la $1^{\text {re }}$ semaine d'incubation. Ces résultats sont analogues aux précédents, si ce n'est le fait, observé aussi pour d'autres cas, de l'existence d'une phase de latence avec les produits les plus jeu-

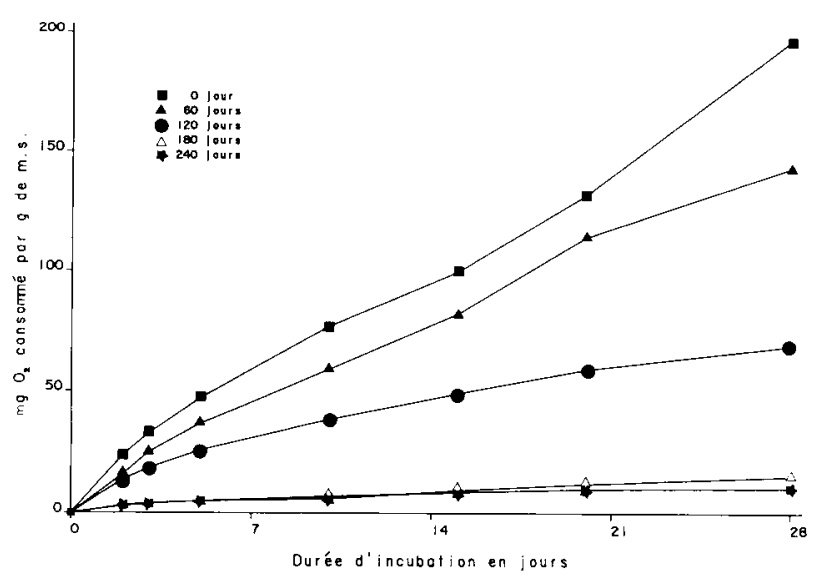

Figure 3

Consommation cumulée d'oxygène pendant $28 j$ de composts d'âges différents (origine des échantillons $A a$; âge exprimé en jours).

28-day cumulated oxygen consumption of different aged composts (sample origin $A a ;$ age in days).

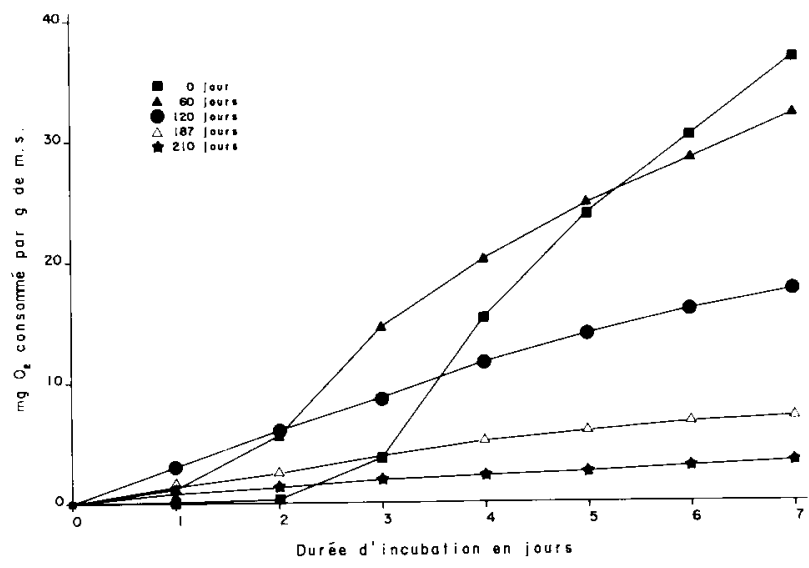

Figure 4

Consommation cumulée d'oxygène pendant $7 j$ de composts d'âges différents (origine des échantillons $C f$; âge exprimé en jours).

7-day cumulated oxygen consumption of different aged composts (sample origin $C f$; age in days).

nes : elle dure ici entre 2 et $3 \mathrm{j}$; nous en avons observé dans certains cas jusqu'à $4 \mathrm{j}$.

\section{Respirométrie de composts dans un sol}

Une $1^{\text {re }}$ expérience d'une durée de 5 mois (fig. 5) intéressant un compost à 3 stades de fermentation : 6, 60 et $120 \mathrm{j}$, montre que l'apport de compost se traduit par une augmentation de l'activité respiratoire du sol et ce, dès le début de l'incubation.

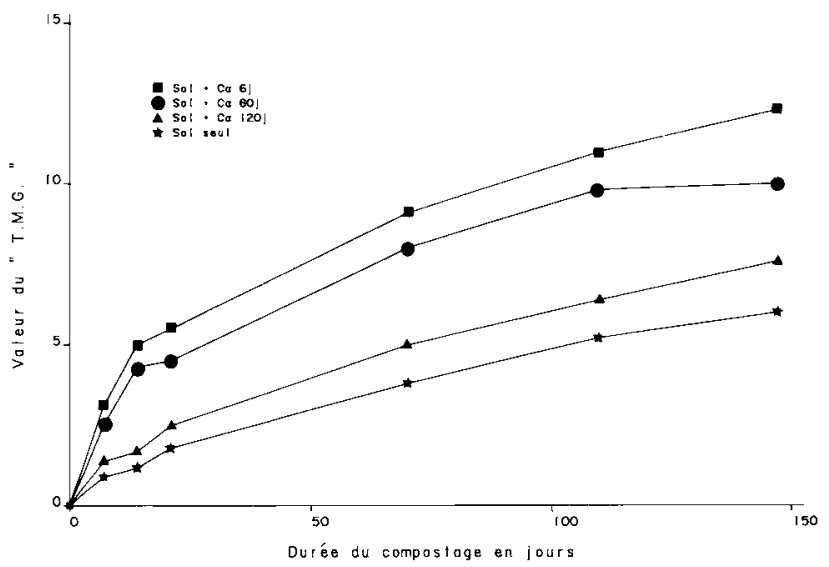

Figure 5

Evolution du taux de minéralisation globale cumulé (T.M.G.) d'un sol enrichi en compost Ca à différents stades de maturité.

Cumulative mineralization ratio (T.M.G.) of a soil amended with compost Ca at different stages of maturation.

Les quantités de $\mathrm{CO}_{2}$ dégagées diminuent avec l'âge du compost : sol + compost de $6 \mathrm{j}>$ sol + compost de $60 \mathrm{j}>$ sol + compost de $120 \mathrm{j}>$ sol seul.

Les estimations indiquent que 50 p. 100 du carbone organique du compost frais est minéralisé au cours des 5 mois d'expérience contre seulement 17 p. 100 pour le compost âgé de $120 \mathrm{j}$. L'accroissement de la respiration du sol, très marqué avec les ordures fraîches, correspond à la transformation des produits facilement biodégradables qui sont d'autant plus 
abondants que le compost a peu évolué. La réduction des composés fermentescibles des ordures au cours du compostage se trouve bien illustrée par l'utilisation de telles techniques respiratoires. Elles permettent, en effet, d'exprimer le degré de stabilisation atteint par les ordures. Enfin, il ressort que ce degré de stabilisation peut être apprécié sur une plus courte période d'incubation, 21 voire $7 \mathrm{j}$, afin de caractériser le comportement d'un plus grand nombre de produits.

Les résultats (fig. 6) représentent, pour quelques produits, l'influence de la durée du compostage sur la respiration au cours d'une incubation de $21 \mathrm{j}$ de mélanges sol + composts. L'analyse de variance montre que la quantité de $\mathrm{CO}_{2}$ dégagée est significativement affectée (niveau 1 p. 100) par l'origine et par l'âge du compost. L'analyse statistique non reproduite ici met également en évidence une interaction origine $\times$ âge, significative. Pour certains composts âgés, les résultats obtenus ne sont pas significativement différents de ceux du sol témoin, traduisant ainsi le fait qu'à partir d'une certaine durée de compostage, les ordures atteignent une stabilité telle qu'elle modifie peu ou pas l'activité respiratoire du sol.

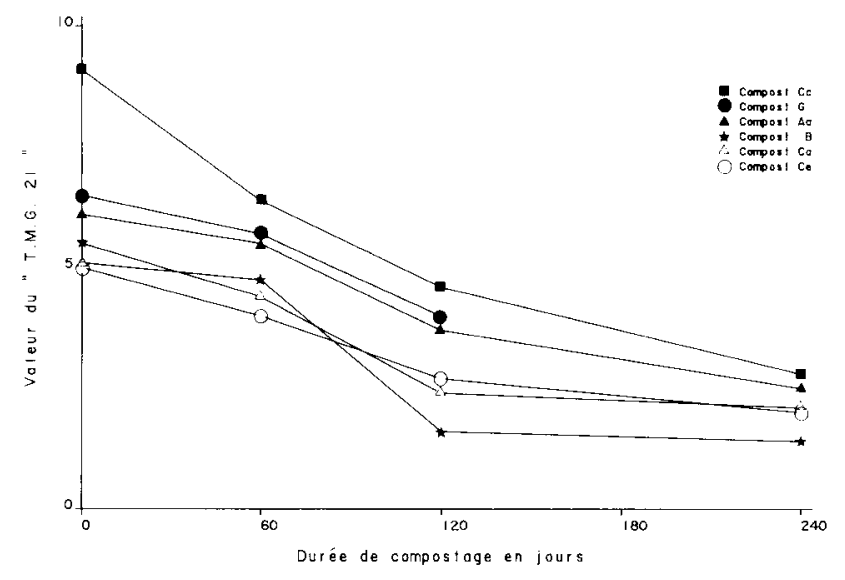

Figure 6

Effet de la durée de compostage et du type d'usine sur la valeur du T.M.G. 21 d'un sol amendé avec des composts d'origines différentes.

Effects of composting duration and type of treatment plant on the T.M.G. 21 value of a soil amended with compost from different origins.

L'interaction indique que, pour des durées équivalentes de compostage, les degrés de stabilisation atteints par les ordures peuvent être très différents selon les usines, confirmant ainsi que la seule connaissance de l'âge d'un compost ne permet pas de juger précisément de son état de stabilisation. Après $21 \mathrm{j}$ d'incubation dans le sol, le taux de décomposition d'ordures compostées est inférieur à 5 p. 100.

\section{CONCLUSION}

L'évolution des ordures ménagères en cours de compostage conduit à des modifications importantes de leur composition.

On observe après $240 \mathrm{j}$ une décroissance marquée des teneurs en carbone organique et une diminution importante $d u$ rapport $\mathrm{C} / \mathrm{N}$. Cependant, si le pourcentage en azote total varie peu, on enregistre néanmoins des pertes sensibles d'azote en valeur absolue. La baisse du taux de matière organique entraîne aussi une chute de l'humidité équivalente. Le $\mathrm{pH}$ par contre s'élève significativement. L'azote se trouve essentiellement sous forme organique, les formes minérales représentant $0,2 \mathrm{p} .100$ après $240 \mathrm{j}$, avec une prédominance de l'azote nitrique. La fraction du phosphore JORET-HÉBERT baisse de moitié, cette évolution étant corrélative de l'élévation du $\mathrm{pH}$.

La caractérisation de certaines formes carbonées des ordures a permis d'étudier leur évolution au cours du compostage. Le stock de composés sous formes dites humifiées s'accroît.

Cette évolution conduit à une stabilisation des composés organiques des composts que l'on peut mettre en évidence lors d'études respirométriques. La respiration de composts seuls ou en mélange avec le sol dépend en effet de la durée de compostage. Elle est fonction aussi de l'origine du produit donc du type de compostage (qualité des ordures ménagères, technologie).

Les technologies variées mises en ouvre au cours du compostage des ordures ménagères aboutissent à des produits présentant des fluctuations notables au niveau de leur composition. Cependant, la faiblesse de l'échantillonnage ne permet pas de réaliser une discrimination nette entre filières de fermentation.

Malgré les pertes en produits carbonés ou azotés, les composts d'ordures ménagères demeurent une source de matière organique intéressante pour l'agriculture. Toutefois, leur utilisation en tant qu'amendements organiques entraîne des règles spécifiques d'utilisation en relation notamment avec le degré d'évolution de leurs constituants organiques. Aussi, est-il nécessaire de pouvoir accéder, par le suivi de certains paramètres analytiques ou biologiques, à la définition de critères de maturité du compost et de proposer des méthodes simples de détermination de cette maturité qui pourraient être utilisées de façon courante dans la pratique.

Recu le 17 avril 1985. Accepté le 11 avril 1986.

\section{REMERCIEMENTS}

Ces travaux ont bénéficié d'aides du Ministère de l'Environnement et du cadre de vie.

\section{RÉFÉRENCES BIBLIOGRAPHIQUES}

Allenspach H., 1969. Détermination du degré de maturité du compost de gadoues. $1^{\text {re }}$ partie - mesure de la diminution d'oxygène. Bull. Inf. du GIROM, Zurich, 35, 330-342.

Anne P., 1945. Sur le dosage rapide du carbone organique des sols, Ann. Agron., 161-172.
Anstett A., 1978. Points de vue de l'agronome quant à l'utilisation de déchets des centres urbains et de l'industrie en agriculture. Génie rural, 11, 28-32.

Basalo C., 1974. Les ordures ménagères en agriculture. T.S.M. L'Eau, 69, 15-23. 
Basalo C., 1978. Le traitement des ordures ménagères. Génie rural, 11, 26-28.

Benistant D., 1978. Caractérisation de la maturité des composts d'ordures ménagères. Mém. fin d'étud., ENITA Dijon, $40 \mathrm{p}$.

Bernard D., 1977. Recherches sur les composts urbains et les déchets broyés. In Ministère de l'Environnement et du Cadre de Vie : Sols et déchets solides, 11, 321-332.

Bouyoucos G. J., 1929. A new, simple and rapid method for determining the moisture equivalent of soils, and the role of soil colloids on this moisture equivalent. Soil Sci., 27, 233-241.

Bremner J. M., 1965. Total nitrogen, inorganic forms of nitrogen. In "Methods of soil analysis", Agronomy, 9 (2), 1149-1237, C. A. Black, D. D. Evans, J. L. White, L. E. Ensminger, F. E. Clark eds, Am. Soc. Agron., Madison (Wisconsin), 771-1572.

Brink R. H., Dubach P., Lynch D. L., 1960. Measurement of carbohydrates in soil hydrolysates with anthrone. Soil Sci., 89, 157166.

Chambolle T., 1978. La politique de gestion des déchets. Génie rural, 11, 5-7.

Chrometzka P., 1968. Détermination de la consommation d'oxygène des composts en voie de maturation. Bull. Inf. du GIROM, Zurich, 33, 253-256.

Colin F., 1978. Recherche de paramètres caractéristiques de la maturité des composts. Etude de l'évolution de la biomasse au cours de la maturation. C. R. fin de contrat 78-142, Ministère de l'Environnement et du Cadre de Vie. Institut de Recherches Hydrologiques, Nancy, $71 \mathrm{p}$.

Dubois M., Gilles K. A., Hamilton J. K., Rebers P. A., Smith F., 1956. Colorimetric method for determination of sugars and related substances. Anal. chem., 28, 350-356.

Duchaufour P., Jacquin F., 1966. Nouvelles recherches sur l'extraction et le fractionnement des composés humiques. Bull. ENSAN, 8, $1-24$.

Germon J. C., Verguet A., 1974. Description d'un respiromètre électrolytique utilisé pour étudier la biodégradabilité d'effluents apportés au sol. Ann. Agron., 25, 195-205.
Golueke C. G., 1977. The biological approach to solid waste management. Compost Sci., 18, 4-9.

Golueke C. G., 1979. Composting : a review of rationale, principles and public health. Compost Sci., 17, 11-15.

Guckert A., 1973. Contribution à l'étude des polysaccharides dans les sols et leur rôle dans les mécanismes d'agrégation. Thèse Doct. ès Sci., Univ. Nancy I, 124 p.

Guckert A., Roger P., Jacquin F., 1968. Contribution à l'utilisation des techniques radioisotopiques pour l'étude de la matière organique du sol. Bull. ENSAN, 10, 69-76.

Inoko A., Miyamatsu K., Sughara K., Harada K., 1979. On some organic constituents of city refuse composts produced in Japan. Soil Sci. Plant Nutr., 25, 225-234.

Inoko A., Harada Y., Sughara K., 1982. Agricultural use of municipal refuse compost with special reference to the degree of maturity. Bull. Natl. Inst. Agric. Sci., séries B, 33, 165-213.

Joret G., Hebert J., 1955. Contribution à la détermination du besoin des sols en acide phosphorique. Ann. Agron., 233-299.

Juste C., Solda P., 1977. Etude des possibilités d'utilisation des composts d'ordures ménagères comme supports de cultures maraîchères. In Ministère de l'Environnement et du Cadre de Vie. Sols et déchets solides, 11, 389-396.

Nicolardot B., 1979. Valorisation agricole des composts d'ordures ménagères. Caractérisation de leur maturité. Accélération de leur évolution dans le sol. Mém. fin d'étud., ENITA Dijon, 59 p.

Pommel B., Bernard D., 1975. Le compost et son marché. Génie rural, 8-9, 255-258.

Salaun M., 1976. Le compostage et le broyage des résidus. Ingénieurs des villes de France, 4, 28-38.

Sugahara K., Inoko A., 1981. Composition analysis of humus and characterization of humic acid obtained from city refuse compost. Soil Sci. Plant Nutrit., 27, 213-224.

Tietjen C., 1975. The potential of composting in developing countries. Compost Sci., 16, 6-7.

Vlamis J., Williams D. E., 1972. Utilization of municipal organic wastes as agricultural fertilizers. Compost. Sci., 13, 26-28. 